\title{
Viral Infections and New Approaches to Their Treatment
}

\author{
Leila Gevorkyan'1, Irina Jashi² \\ ${ }^{1}$ Tbilisi Central Hospital, Tbilisi, Georgia \\ ${ }^{2}$ Institute of Clinical Cardiology, Tbilisi, Georgia \\ Email: leila.gev@hotmail.com
}

How to cite this paper: Gevorkyan, L. and Jashi, I. (2020) Viral Infections and New Approaches to Their Treatment. Open Access Library Journal, 7: e6384. https://doi.org/10.4236/oalib.1106384

Received: April 30, 2020

Accepted: June 8, 2020

Published: June 11, 2020

Copyright (c) 2020 by author(s) and Open Access Library Inc.

This work is licensed under the Creative Commons Attribution International License (CC BY 4.0).

http://creativecommons.org/licenses/by/4.0/

\begin{abstract}
The article discusses a new version of the fight against viral infections. The use of certain bacterial enzymes that can possibly be used to destroy viral RNA and DNA is discussed. The proposed hypothesis is based on the available data regarding the struggle of bacteria with their viruses that infect themselves. Since opportunistic bacteria are capable of producing certain enzymes, in particular staphylococcal DNase and other bacterial restriction enzymes, they can cope with their own viruses, and may also be used to fight viruses that infect the human body.
\end{abstract}

\section{Subject Areas}

Viral Infections

\section{Keywords}

Viruses, Conditionally Pathogenic Bacteria, Staphylococci, Deoxyribonuclease, Bacterial Restrictase

Viral infections in the annual number of incidence rank first in the world. And mortality due to complications from viral infections, according to statistics, is several hundred thousand annually. Despite all efforts, no cure for viruses has yet been found. And even in the fight against the flu virus, all hope remains on the body itself, which at least three days, but still the virus defeats. But the medicine that would help the body recover on the second day does not yet exist, not to mention the cases when a viral infection leads to serious complications and even death.

The article presents theoretical assumptions about the possible methods of fight against viral infections based on our knowledge of infectious diseas- 
es-viral, bacterial, and the body's immune system and the corresponding conclusions based on this knowledge.

Everyone knows that infection with a virus causes an immune response in animals. The immune response can also be triggered by vaccines that develop artificially acquired immunity to a specific viral infection. Some viruses, including those that cause AIDS, HPV infection, and viral hepatitis, avoid these immune reactions and lead to chronic infections. Several antiviral drugs have been developed. Until now, the main antiviral control agents remain vaccines that cause artificial antiviral immunity to certain viruses.

But what if we take a slightly different look at this problem and try to answer a few questions?

Why with viral infections if we give the patient an antibiotic, the body temperature rises? After all, it is known that in order for the patient to recover from influenza or another viral infection, the immune system (lymphocytes, reticular tissue, macrophages, leukocytes) must be turned on, which will produce antibodies within a few days, using which will neutralize the virus. And the patient will recover. And if we make a diagnosis incorrectly, taking a viral infection as a bacterial infection, prescribe an antibiotic, then the body temperature will rise or stay at a certain high level, preventing the body from recovering from a viral infection. Why do antibiotics stop the body from fighting viruses? After all, antibiotics kill bacteria. In particular, with a viral infection, they will kill opportunistic bacteria in the body. As you know, antibiotics do not affect the formation of antibodies, i.e. do not inhibit lymphocytes, reticular tissue. Perhaps in very high doses and with prolonged treatment, they can lead to leukopenia, through the suppression of the myelocytic clone of hematopoietic cells.

If all this is true, then it turns out that by suppressing the conditionally pathogenic flora we prevent the patient from recovering from a viral infection. The viral infection must be defeated by the immune system. The question arises, why when prescribing antibiotics for a viral infection the immune system is suppressed and the body cannot control the virus? Is it really not just the immune system that takes part in the fight against the virus? What else? Maybe-the bacteria themselves?

The development of a bacterial infection as a complication of the virus is explained by the suppression of the body's immune system by the virus, which leads to the activation and reproduction of the opportunistic flora that normally inhabits the skin and mucous membranes of the body. In this case, the question arises, if a viral infection suppresses the body's immune system, how does the latter control the virus? What actually happens-suppression of the immune system or its activation? After all, in the end, the patient recovers from a viral infection. And if the virus suppresses the immune system, against this background a bacterial infection develops (for example, bacterial pneumonia), we begin to treat such patients with antibiotics and the patient recovers from pneumonia. And where does the virus go? Is the patient cured of both bacterial 
and viral infections? If the virus suppressed the immune system, caused a bacterial infection, antibiotics killed the bacteria, but not the viruses, then what killed the viruses?

Consider what happens to the skin or mucous membranes when they are injured. With a skin injury, its integrity is broken and if the skin is not treated with an antiseptic in time, then the inflammatory process starts at the site of damage with the formation, ultimately, of a pustule-an abscess. The pus, of course, is the result of penetration from the damaged skin of bacteria into the tissue and the fight against them of the body's defenses-leukocytes. When viruses enter the body, they do not invade through injured cover (although such a transmission route is not excluded, we consider the airborne transmission route, for example), the virus penetrates into the cell without damaging the cell membrane initially, and, therefore, the cover of tissue. The virus, invading the cell, and then into its nucleus, uses it for its reproduction and leaves the cell, in some cases destroying it (both the integrity of the cell membrane and the entire cell), in others-it does not. The virus, having multiplied, is introduced into other cells, which, ultimately, leads to the response of the body in the form of an inflammatory process in this area of the tissue. But as a result of the inflammatory reaction, as well as the presence of destroyed cells infected with the virus, the integrity of the mucous membrane of a given tissue site is broken, which allows the bacteria inhabiting this area to penetrate into the tissue and lead to any changes in it. Is it possible to assume that a certain struggle will take place between bacteria and viruses, which will lead to the victory of bacteria over viruses and the recovery of the patient, most often, without any complications?

Consider two cases of viral infection-chickenpox and shingles. Both of these diseases are caused by the same virus-a virus from the herpes group. It is known that with chickenpox the epithelial effects of the virus come to the fore, and with herpes zoster-its neurotrophic properties. The patient recovers from chickenpox in a few days, and with shingles, the disease lasts longer, the virus can capture more and more new tissue sites, and even after recovery can recur with a decrease in the body's defenses (suppression of the immune system). Let us try to explain these two diseases as follows: in case of chickenpox, when the virus occupies the epithelial integument, the conditionally pathogenic bacterial flora enters the fight against viruses, which can quickly overcome the viral infection and lead the patient to a fast recovery. And with shingles, viruses penetrate deep into the body and persist in tissues, for example, in the nervous, where there are no conditionally pathogenic bacteria, as a result of which viruses accompany the body all their lives, often manifesting themselves in individuals who have undergone various influences that weaken their immune system.

In acute respiratory viral infections with rhinitis, pharyngitis, tracheitis, when viruses are present in the mucous membranes of the upper respiratory tract, which is rich in opportunistic bacteria, the immune system, for some reason, it is quite easy to control the infection. If the virus overcomes this barrier (epithelial) 
and penetrates into deeper tissues, where the conditionally pathogenic flora is absent, and causes, for example, viral pneumonia, then the immune system "begins to limp" and the patient recovers from viral pneumonia, not complicated by bacterial, very hard. Similarly, with all childhood viral infections, which are mainly epithelial, the child's body can easily control them. With their complications, when the virus penetrates deeper, causing myocarditis, encephalitis, etc., i.e. pathological processes of non-bacterial etiology, for some reason, neither the antibodies that should have been developed by now, nor the white blood cells, nor macrophages can fight the virus. In addition, note that children successfully cope with childhood viral infections than adults with them. Can the latter be explained by the fact that the number of bacteria on the epithelial integument (taking into account the ratio of the surface area of the body to its volume) is much larger in children than in adults, which cannot be said about the protective factors of the immune system?

If we assume that all of the above is possible, then given the large variety of conditionally pathogenic bacteria (staphylococci, streptococci, pneumococci, enterococci, E. coli) [1] [2] [3], as well as candida, etc., the question arises as to which of them may be pathogenic for body cells affected by a virus?

To answer this question, we recall that post-influenza pneumonia is more often of bacterial etiology (staphylococcal, streptococcal, pneumococcal, etc.), it is also viral. Among bacteria, according to statistics, staphylococcal flora most often acts as the etiological factor of post-influenza pneumonia. Let's make some comparisons between different bacteria. For example, it is known that staphylococci synthesize an enzyme called coagulase, which leads to blood coagulation, protecting bacteria from phagocytosis [4]. Streptococci and related pneumococci and enterococci, in contrast to staphylococci, do not have coagulase-synthesizing ability, thereby they are less protected from the body's immune system. Further, staphylococci quickly develop resistance to antibiotics (penicillin), which cannot be said about other bacteria. Streptococci are more sensitive to various antibiotics (penicillin, tetracycline drugs, erythromycin, chloramphenicol) [5]. Antibiotic resistance is also confirmed by the development of staphylococcal enterocolitis in patients receiving broad-spectrum antibiotics (tetracycline or a combination of antibiotics), the prescribing of which leads to the disappearance of the normal intestinal flora, while staphylococci remain not killed [5] [6] [7] [8]. Finally, immunity in staphylococcal diseases is very weak. Streptococcal infections are characterized by the development of both antitoxic and antimicrobial immunity (for example, with scarlet fever).

It turns out that among opportunistic bacteria, the most resistant is staphylococcus. And to fight against viral infections, taking into account their diversity and mutational abilities, it is apparently easier for a stronger bacterium.

So, suppose staphylococcus can fight the virus. How can endogenous staphylococcus kill a cell infected with a virus?

Let's remember that plants, fungi, protists, bacteria are also affected by virus- 
es. Despite the fact that viruses can have a harmful effect on them, they still have quite sophisticated and effective methods of protection against viruses. For example, in plants, the most effective mechanism is the presence of the so-called (R) resistance gene. Each R-gene is responsible for resistance to a particular virus and causes the death of cells adjacent to the affected, which can often be seen with the unaided eye as large spots. This stops the infection from spreading [9] [10].

In penicillin fungi, antiviral activity has been established, which is caused by the induction of interferon of double-stranded RNA from viruses that infect fungi.

As for bacteria, the main mechanism for protecting bacterial cells from bacteriophages is the formation of enzymes that destroy foreign DNA. These enzymes, called restriction endonucleases, "cut" the viral DNA injected into the cell. Bacteria also use a system called CRISPR (clustered regularly interspaced short palindromic repeats) that stores information about the genomes of viruses that the bacterium has previously encountered. This system provides acquired immunity of the bacterial cell [11] [12] [13].

Restriction endonucleases-restrictases that are produced by many bacteria, including E. coli, are a group of enzymes belonging to the class of hydrolases that catalyze the hydrolysis of nucleic acids. These enzymes are found in bacteria and archaea and provide a defence mechanism against invading viruses. Inside a prokaryote, the restriction enzymes selectively cut up foreign DNA in a process called restriction digestion. Currently, more than three thousand restriction endonucleases have been isolated, many of which are used in genetic engineering [14] [15] [16] [17] [18].

In addition, Staphylococcus aureus produces various enzymes, such as coagulase, which coagulates the plasma and covers the bacterial cell, possibly protecting it from phagocytosis, hyaluronidase, which breaks down hyaluronic acids and helps the spread of bacteria, beta-lactamase, etc. [4]. Staphylococcus aureus also produces deoxyribonuclease, which destroys DNA [12] [19] [20] [21].

Deoxyribonuclease produced by Staphylococcus aureus is an enzyme that catalyzes the hydrolytic cleavage of phosphodiester bonds in the main DNA strand, thereby destroying it. DNase enzymes are used, for example, in cystic fibrosis: leukocytes that accumulate in the mucus are destroyed, secrete DNA, which enhances the stickiness of the mucus, and in this case, DNase enzymes are used that break down the DNA and facilitate the release of mucus [19] [20] [21].

Perhaps, similarly to the mechanisms described above, the use of restrictase or DNAse for patients with viral infection will lead to the cleavage of the DNA of the body cells that have been infected by the virus, or of the RNA or DNA viruses themselves.

Suppose that, in fact, with a viral infection, the activation of conditionally pathogenic staphylococcal bacteria will occur, their reproduction, and, possibly, the formation of staphylococcal enzymes that will be included in the fight against viruses. Perhaps after this fight, when the viruses are completely de- 
stroyed by staphylococci or their enzymes, these bacteria will either cease to multiply and the patient will completely recover from the viral infection without complications, or staphylococci, after fighting the viruses, completely fighting the latter, will continue to multiply from the conditionally pathogenic will turn into a pathogenic flora, causing a bacterial infection that will require the inclusion of antibiotics in the course of treatment.

Why staphylococci in such cases can continue their reproduction?

Suppose that in a sick body (meaning a patient with a viral infection), conditionally pathogenic staphylococci are enough to completely overcome all viruses of the infected organism themselves or by their enzymes. That means the number of staphylococci in the body is in sufficient quantity to control all viruses that have affected this body. And if staphylococci in any body are not in the required quantity to destroy all viruses, then staphylococci begin to multiply in order to reach such a level of their quantity that is necessary to fight viruses. Theoretically, this is acceptable if we recall that with a viral infection in most cases there is a picture of leukopenia. That is the viral infection, suppressing the body's immune system, leads to a decrease in the number of leukocytes in it, which may favor the reproduction of opportunistic bacteria. It is possible that such reproduction of staphylococci will turn out to be a trigger mechanism, which will lead to the formation of such a quantity that will exceed their normal upper limit and will become a pathogenic flora for the body.

Summarizing all of the above, it can be assumed that not only the immune system is involved in the fight against viral infections. Probably, opportunistic flora plays an important role in this. Therefore, maybe staphylococcal DNases or other bacterial restriction enzymes can be used for acute respiratory viral infections, and possibly for other viral infections, including those that evade the body's immune response and accompany the body chronically. As for viral infections accompanied by diarrhea (so-called intestinal infections), restriction enzymes produced by Escherichia coli may also be useful.

Thus, staphylococcal DNases and other bacterial restriction enzymes, as in the case of bacteria fighting their own viruses, may prevent the multiplication of viruses in the body of a patient with a viral infection and lead the patient to a quick recovery.

\section{Conflicts of Interest}

The authors declare no conflicts of interest regarding the publication of this paper.

\section{References}

[1] Kloos, W.E. (1980) Natural Populations of the Genus Staphylococcus. Annual Review of Microbiology, 34, 559-592.

https://doi.org/10.1146/annurev.mi.34.100180.003015

[2] Kluytmans, J., van Belkum, A. and Verbrugh, H. (1997) Nasal Carriage of Staphylococcus aureus. Epidemiology, Underlying Mechanisms, and Associated Risks. 
Clinical Microbiology Reviews, 10, 505-520.

https://doi.org/10.1128/CMR.10.3.505-520.1997

[3] Tong, S.Y., Davis, J.S., Eichenberger, E., Holland, T.L. and Fowler, V.G. (2015) Staphylococcus aureus Infections: Epidemiology, Pathophysiology, Clinical Manifestations, and Management. Clinical Microbiology Reviews, 28, 603-661.

https://doi.org/10.1128/CMR.00134-14

[4] Ryan, K.J. and Ray, C.G., Eds. (2004) Sherris Medical Microbiology. 4th Edition, McGraw-Hill, New York.

[5] Kazantsev, A.P. and Matkovsky, V.S. (1986) Infectious Disease Handbook. 3rd Edition, Medicine, Moscow.

[6] Dinges, M.M., Orwin, P.M. and Schlievert, P.M. (2000) Exotoxins of Staphylococcus aureus. Clinical Microbiology Reviews, 13, 16-34.

https://doi.org/10.1128/CMR.13.1.16

[7] Jarraud, S., Peyrat, M.A., Lim, A., Tristan, A., Bes, M., Mougel, C., Etienne, J., Vandenesch, F., Bonneville, M. and Lina, G. (2001) egc, a Highly Prevalent Operon of Enterotoxin Gene, Forms a Putative Nursery of Superantigens in Staphylococcus aureus. Journal of Immunology, 166, 669-677.

https://doi.org/10.4049/jimmunol.166.1.669

[8] Becker, K., Friedrich, A.W., Lubritz, G., Weilert, M., Peters, G. and Von Eiff, C. (2003) Prevalence of Genes Encoding Pyrogenic Toxin Superantigens and Exfoliative Toxins among Strains of Staphylococcus aureus Isolated from Blood and Nasal Specimens. Journal of Clinical Microbiology, 41, 1434-1439.

https://doi.org/10.1128/JCM.41.4.1434-1439.2003

[9] Rybicki, E.P. (2015) A Top Ten List for Economically Important Plant Viruses. Archives of Virology, 160, 17-20. https://doi.org/10.1007/s00705-014-2295-9

[10] Dinesh-Kumar, S.P., Tham, W.-H. and Baker, B.J. (2000) Structure-Function Analysis of the Tobacco Mosaic Virus Resistance Gene N. Proceedings of the National Academy of Sciences of the United States of America, 97, 14789-14794. https://doi.org/10.1073/pnas.97.26.14789

[11] Bickle, T.A. and Kruger, D.H. (1993) Biology of DNA Restriction. Microbiology and Molecular Biology Reviews, 57, 434-450. https://doi.org/10.1128/MMBR.57.2.434-450.1993

[12] Barrangou, R., Fremaux, C., Deveau, H., et al. (2007) CRISPR Provides Acquired Resistance against Viruses in Prokaryotes. Science, 315, 1709-1712. https://doi.org/10.1126/science.1138140

[13] Brouns, S.J., Jore, M.M., Lundgren, M., et al. (2008) Small CRISPR RNAs Guide Antiviral Defense in Prokaryotes. Science, 321, 960-964. https://doi.org/10.1126/science.1159689

[14] Roberts, R.J., Vincze, T., Posfai, J. and Macelis, D (2007) REBASE-Enzymes and Genes for DNA Restriction and Modification. Nucleic Acids Research, 35, D269-D270. https://doi.org/10.1093/nar/gkl891

[15] Roberts, R.J. (1976) Restriction Endonucleases. CRC Critical Reviews in Biochemistry, 4, 123-164. https://doi.org/10.3109/10409237609105456

[16] Kessler, C. and Manta, V. (1990) Specificity of Restriction Endonucleases and DNA Modification Methyltransferases: A Review (Edition 3). Gene, 92, 1-240. https://doi.org/10.1016/0378-1119(90)90486-B

[17] Pingoud, A., Alves, J. and Geiger, R. (1993) Chapter 8: Restriction Enzymes. In: Burrell, M., Ed., Enzymes of Molecular Biology, Humana Press, Totowa, NJ, 107-200. 
[18] Kobayashi, I. (2001) Behavior of Restriction-Modification Systems as Selfish Mobile Elements and Their Impact on Genome Evolution. Nucleic Acids Research, 29, 3742-3756. https://doi.org/10.1093/nar/29.18.3742

[19] Bowen, R.A., Austgen, L. and Rouge, M. (2004) Restriction Endonucleases and DNA Modifying Enzymes (2017) Nucleases: DNase and RNase, Biotechnology and Genetic Engineering. http://arbl.cvmbs.colostate.edu/hbooks/genetics/biotech/enzymes/nucleases.html

[20] Rahman, N. (2011) Intrapleural Use of Tissue Plasminogen Activator and DNase in Pleural Infection. The New England Journal of Medicine, 365, 518-526. https://doi.org/10.1056/NEJMoa1012740

[21] Ninfa, A.J., Ballou, D.P. and Benore, M. (2010) Fundamental Laboratory Approaches for Biochemistry and Biotechnology. 2nd Edition, John Wiley \& Sons, Hoboken, 234. 